\title{
Classification with Maximum Entropy Modeling of Predictive Association Rules
}

\author{
Hieu X. Phan ${ }^{1}$, Minh L. Nguyen ${ }^{1}$, S. Horiguchi ${ }^{2}$, Bao T. Ho ${ }^{1}$, and Y. Inoguchi ${ }^{1}$ \\ 1 Japan Advanced Institute of Science and Technology, \\ 1-1, Asahidai, Tatsunokuchi, Ishikawa, 923-1211, Japan \\ \{hieuxuan, nguyenml, inoguchi, bao\}@jaist.ac.jp \\ 2 Tohoku University, Aoba 6-3-09 Sendai, \\ 980-8579, Japan \\ susumu@ecei.tohoku.ac.jp
}

\begin{abstract}
This paper presents a new classification model in which a classifier is built upon predictive association rules (PARs) and the maximum entropy principle (maxent). In this model, PARs can be seen as confident statistical patterns discovered from training data with strong dependencies and correlations among data items. Maxent, on the other hand, is an approach to build an estimated distribution having maximum entropy while obeying a potentially large number of useful features observed in empirical data. The underlying idea of our model is that PARs have suitable characteristics to serve as features for maxent. As a result, our classifier can take advantage of both the useful correlation and confidence of PARs as well as the strong statistical modeling capability of maxent. The experimental results show that our model can achieve significantly higher accuracy in comparison with the previous methods.
\end{abstract}

\section{Introduction}

Building efficient classifiers is a major task of machine learning research. Given a training set of data instances together with their class labels, the classification task is to induce a classifier that can predict labels for unseen instances. Traditional methods for this task have followed various approaches like rule learning [5] [6], decision trees [21, and statistical models (e.g., Naive Bayes [10]).

With the emergence of high-performance data mining techniques for largescale databases, recent studies have shown that associative classification, using confident PARs discovered from training data, is competitive with the traditional methods in terms of both accuracy and scalability. The associative classification methods, such as CBA [16], CAEP [9], CMAR [1], and ART [3], build classifiers based on confident PARs whose consequent is a class label. Since the number of PARs is usually large, these methods attempt to select a subset by pruning the original set according to some heuristic assumption. The selected rules are then used to predict labels for new instances in a number of ways: ranking and searching for the most appropriate rule (CBA), computing an aggregated differentiating score based on emerging patterns to determine the most suitable class 
(CAEP), analyzing to select a small subset of the most related rules and making a collective classification decision on that subset (CMAR), and classifying with a decision list of PARs which is built by using Occam's razor as an inductive bias (ART), i.e., giving a higher priority to simpler hypotheses.

This presents a new strategy to build a classifier upon PARs and maxent. In our approach, those PARs are treated as features/constraints of the maxent distribution, and each of them is associated with a learnable weight. Training the classifier is to find the weight values that maximize the entropy of the above distribution. Once trained, the classifier can predict a label for each new data instance by summing over the learned weights of PARs belonging to different class labels in order to determine the most appropriate one. The main motivation of our approach can be summarized as follows.

- PARs are confident facts with highly correlated conjunctions of data items at their antecedent. And they, in a sense, can be viewed as complex statistic patterns hidden in empirical data. Thus, incorporating those PARs into a statistical model means that we can project the original feature space into a richer one where PARs can cover and control various classifying situations.

- Maxent is a powerful statistical model that can include millions of overlapping, non-independent features/constraints, and thus provides enough room for a potentially large number of PARs. Further, the maxent distribution is totally consistent with the maximum likelihood distribution 2. Thus, our classifier relies not only on confident implications, but also on a solid statistical principle. Also, this principle can be seen as an inductive bias for inducing our classifier because maxent follows Occam's razor to choose the least complex distribution among the others that satisfy empirical constraints.

- Unlike other rule-based classifiers, our method predicts class labels for new instances based on multiple PARs w.r.t the global interaction among them and the aggregation of their weights. Obviously, this helps avoid prediction based on a single PAR, which sometimes leads to biased decisions. This is very important because predicting based on a high-ranked rule is not always optimal for hard instances that may exist in ambiguous or unbalanced data.

The remainder of the paper is organized as follows. Section 2 mainly presents our classification model. Section 3 gives the experimental results and some discussion. Finally, conclusions are given in Section 4 .

\section{The Proposed Classification Model}

\subsection{Conditional Maximum Entropy}

Although maxent has a long history and a wide range of applications, this paper confines this principle to the problem of building a model that can best describe empirical data. Intuitively, maxent models all that is known and assume nothing about what is unknown [2]. In other words, given a collection of facts, choose a model consistent with all the facts, but otherwise as uniform as possible. Maxent 
has been successfully applied to many natural language processing tasks, e.g. machine translation [2, named entity recognition [4], and POS tagging 22.

Given a training dataset $D=\left\{\left(o^{i}, l^{i}\right)\right\}_{i=1}^{n}$ in which $o^{i}$ is the $i^{\text {th }}$ data instance and $l^{i}(\in \mathcal{L})$ is its class label. Conditional maxent is a conditional distribution $P(l \mid o)$ - the conditional probability of having the class label $l$ given the instance $o$. To train maxent model, experimenters have to determine significant features in the training data and integrate them into the model in terms of constraints. Maxent features have the form of two-argument function $f:(o, l) \rightarrow R$ below:

$$
f_{<c p, l^{\prime}>}(o, l)=\left\{\begin{array}{l}
1 \text { if } l=l^{\prime} \text { and } c p(o)=\text { TRUE } \\
0 \text { otherwise }
\end{array}\right.
$$

where $l^{\prime}$ is a particular label and $c p$ is a particular context predicate indicating a useful property of $o$. The maxent model is consistent with $D$ w.r.t every feature $f_{i}$ by satisfying constraints like $E\left(f_{i}\right)=F\left(f_{i}\right)$ : the expected value of $f_{i}$ w.r.t $D$ is equal to the expected value of $f_{i}$ w.r.t $P(l \mid o)$. The maxent model is the distribution having the highest entropy while satisfying those constraints. Using the Lagrange multipliers method and theory of constrained optimization, Pietra et al. 20] proved that maxent model is unique and has the exponential form.

$$
P_{\lambda}(l \mid o)=\frac{1}{Z_{\lambda}(o)} \exp \left(\sum_{i=1} \lambda_{i} f_{i}(o, l)\right),
$$

where $\lambda_{i}$ is the weight associated with $f_{i}$, and $Z_{\lambda}(o)$ is the normalizing factor. Maxent is trained by setting the weight set $\left\{\lambda_{1}, \ldots\right\}$ to maximize the entropy $H\left(P_{\lambda}\right)$. The maxent model was often trained using GIS [7. Recent studies [19] have shown that quasi-Newton methods like L-BFGS [18] are more efficient than the others. Once trained, the model will be used to predict labels for unseen data. Given a new instance $o$, the predicted label of $o$ is $l_{o}=\operatorname{argmax}_{l \in \mathcal{L}} P_{\lambda}(l \mid o)$.

\subsection{Mining Predictive Association Rules from Training Data}

Let $\mathcal{A}=\left(A_{1}, \ldots, A_{m}\right)$ be a data schema of $m$ attributes in which $A_{i}$ is either discrete or continuous. For any continuous attribute, its domain can be divided into non-overlapping intervals by using discretization methods [11, [12] so that all attributes can be treated uniformly as discrete. Let $\mathcal{L}=\left\{l_{1}, \ldots, l_{q}\right\}$ be the class attribute having $q$ class labels. Given a training data $D=\left\{\left(o^{i}, l^{i}\right)\right\}_{i=1}^{n}$ of $n$ data instances in which each $o^{i}=\left(a_{1}^{i}, \ldots, a_{m}^{i}\right)$ follows the above schema and $l^{i}$ is a label associated with $o_{i}$. PAR, discovered from $D$, is a special type of association rule [1] and has the form:

$$
\left\{\left[A_{i_{1}}=a_{i_{1}}\right] \wedge\left[A_{i_{2}}=a_{i_{2}}\right] \wedge \ldots \wedge\left[A_{i_{k}}=a_{i_{k}}\right]\right\} \Rightarrow l,
$$

where the PAR antecedent is a conjunction of $k$ attribute-value pairs and the consequent is a class label. The support of a PAR is the number of instances in $D$ that match both its antecedent and its consequent. The confidence of a PAR is the conditional probability that an instance matches the consequent given that it matches the antecedent. Confident PARs are those whose support and 
confidence are greater than or equal to given minimum support (minsup) and minimum confidence (minconf), respectively.

We use the FP-growth algorithm [14, a very efficient frequent pattern mining technique, to mine association rules [1. Then, we filter confident PARs from outputs of FP-growth. Unlike the previous methods, we apply different minconf thresholds $\left(\operatorname{mincon} f_{1}, \ldots, \operatorname{mincon}_{k}\right)$ corresponding to different antecedent lengths. This is because of an important observation that short rules usually have smaller confidence but are very useful for generalization while long rules have higher confidence but often are too specific.

\subsection{MEPAR: Maximum Entropy Modeling of PARs}

This section describes how to incorporate confident PARs into the maxent model to build our classifier - MEPAR. As mentioned earlier, maxent features are twoargument functions $f_{<c p, l^{\prime}>}(o, l)$. For example, in the problem of determining part-of-speech (POS) labels (e.g., noun, verb, adjective, etc.) for English words using a maxent model, we should include the useful fact that "if a word $o$ ends with a suffix tive, it is very likely that the word $o$ is an adjective". We interpret this fact as a maxent feature $f_{<\text {suffix_tive, } \operatorname{adj}>}(o, l)$. Obviously, this feature is useful for predicting POS labels for new words because it relies on a confident statistic from empirical data. Interestingly, confident PARs are also confident facts in training data, and thus can naturally be incorporated into the maxent model as normal maxent features. Given a confident PAR $r:\left\{\left[A_{i_{1}}=a_{i_{1}}\right] \wedge \ldots \wedge\right.$ $\left.\left[A_{i_{k}}=a_{i_{k}}\right]\right\} \Rightarrow l^{r}$, its corresponding maxent feature can be written as,

$$
f_{<\operatorname{match}_{-}\{\ldots\}, l^{r}>}(o, l)= \begin{cases}1+\operatorname{conf}(r) & \text { if } l=l^{r} \text { and "o matches } \\ & \left\{\left[A_{i_{1}}=a_{i_{1}}\right] \wedge \ldots \wedge\left[A_{i_{k}}=a_{i_{k}}\right]\right\} " \\ 0 & \text { otherwise }\end{cases}
$$

In (41), a feature is active $(=1+\operatorname{conf}(r))$ if the label $l$ of $o$ is matching with $l^{r}$ and $o$ holds the antecedent of $r$. Training maxent is very complicated in such a way that features interact with each other to yield an optimal estimated distribution over the training data. Thus, the learned weight of a PAR not only reflects how important the PAR is but also w.r.t the global optimum. One important observation is that the maxent model relies mainly on the occurrence frequency of its features and ignores feature confidence. In our model, we provide a priori for PARs by adding their confidence $(\operatorname{conf}(r))$ to their corresponding feature values. After being trained, MPEAR can be used to predict class labels for unseen instances: given a new instance $o^{j}$, its predicted label $l^{j}$ will be:

$$
\begin{aligned}
& l^{j}=\operatorname{argmax}_{l \in \mathcal{L}} P_{\lambda}\left(l \mid o^{j}\right)
\end{aligned}
$$

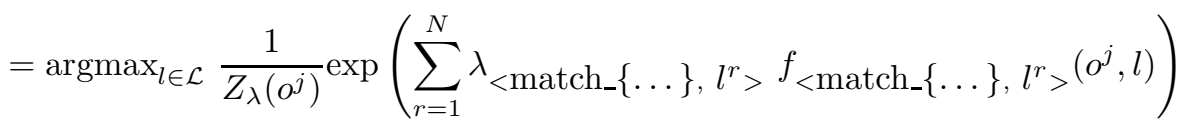

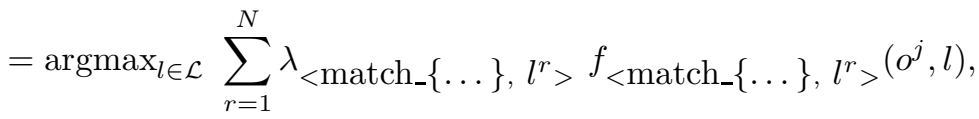




\section{Experiments}

\subsection{Experimental Environments, Parameters, and Evaluation}

The experiments were performed using our $\mathrm{C} / \mathrm{C}++$ implementation of maxent (FlexME: www.jaist.ac.jp/ hieuxuan/flexcrfs/flexcrfs.html\#FlexME). PARs were discovered by using an efficient implementation of FP-growth by B. Goethals.

We used two groups of datasets from UCI ML Repository [15] as the experimental data. The first group includes 26 datasets which were tested with CBA [16 and CMAR 17] and the second group contains 13 datasets which were tested with ART 3. Data discretization was done by using the entropy-based discretization method in [1]. The code is taken from MLC ++ library 13 .

All experimental parameters and results of learning methods for the first data group were derived from CMAR [17]; and those of the learning methods for the second data group were taken from ART [3].

We discovered all PARs with the antecedent length $k=1, \ldots, 5$ with mincon $_{1}$ $=40 \%$, mincon $_{2}=70 \%$, mincon $_{3}=80 \%$, minconf $_{4}=90 \%$, mincon $_{5}=95 \%$, respectively. The minsup is $1 \%$. All the experimental results, including ours, were obtained from 10-CV (ten fold cross-validation) tests.

\subsection{Experimental Results}

For each dataset, we performed 10-CV test, and in each $\mathrm{CV}$-fold we trained MEPAR for 50 L-BFGS iterations and then chose the highest accuracy to calculate the 10-CV accuracy. We observed that MEPAR models for UCI datasets converged around the first 20 L-BFGS iterations.

Table 1 shows the results of five methods (C4.5 [21, CBA [16], CAEP 9], CMAR [17, and ours - MEPAR) for the first data group. The first column is the list of 26 datasets; the second is the number of records; the third is the number of attributes; and the fourth is the number of class labels. The results for $\mathrm{C} 4.5$, CBA, and CMAR were taken from [17]; the results for CAEP were taken from [9] in which the results of several datasets are ignored because of their omission from the original paper. The ninth is the accuracy of our model, MEPAR, and the last column is the accuracy of normal maxent (i.e., without PARs). For each row, the highest accuracy is printed using italics.

In the first data group, MEPAR outperformed the other methods on 24 of 26 datasets including both the largest dataset (Waveform, 5,000 records) and the smallest one (Labor, 57 records). MEPAR performs well on datasets with a large number of attributes. This is because these datasets usually generate a large number of PARs with rich dependencies among data items, very useful for the MEPAR model. For those with small number of attributes, MEPAR obtains accuracies similar to the others. The average accuracy of MEPAR is $88.39 \%$, significantly higher than those of CMAR, CBA, C4.5, and normal maxent.

Table 2 shows the results of six methods (ART 3], C4.5 [21], RIPPER ${ }_{k=2}$ [6], CN2 [5], Naive Bayes [10, and ours - MEPAR) for the second data group. The experimental results of ART, C4.5, RIPPER, CN2, and Naive Bayes were 
Table 1. Accuracy comparison: C4.5, CBA, CAEP, CMAR, and MEPAR

\begin{tabular}{|l|r|r|r|c|c|c|c|c|c|}
\hline Datasets & \#Rec & \#Attr & \#Cls & C4.5 & CBA & CAEP & CMAR & MEPAR & N.Maxent \\
\hline \hline Anneal & 898 & 38 & 6 & 94.8 & 97.9 & N/A & 97.3 & 98.9 & 97.5 \\
\hline Austra & 690 & 14 & 2 & 84.7 & 84.9 & 86.2 & 86.1 & 88.4 & 88.0 \\
\hline Auto & 205 & 25 & 7 & 80.1 & 78.3 & N/A & 78.1 & 83.5 & 83.5 \\
\hline Breast & 699 & 10 & 2 & 95 & 96.3 & 97.3 & 96.4 & 97.8 & 97.2 \\
\hline Cleve & 303 & 13 & 2 & 78.2 & 82.8 & 83.3 & 82.2 & 85.3 & 82.2 \\
\hline Crx & 690 & 15 & 2 & 84.9 & 84.7 & N/A & 84.9 & 84.6 & 83.0 \\
\hline Diabetes & 768 & 8 & 2 & 74.2 & 74.5 & N/A & 75.8 & 77.6 & 77.6 \\
\hline German & 1000 & 20 & 2 & 72.3 & 73.4 & 72.5 & 74.9 & 76.6 & 73.5 \\
\hline Glass & 214 & 9 & 7 & 68.7 & 73.9 & N/A & 70.1 & 81.9 & 81.7 \\
\hline Heart & 270 & 13 & 2 & 80.8 & 81.9 & 83.7 & 82.2 & 84.8 & 84.8 \\
\hline Hepatitis & 155 & 19 & 2 & 80.6 & 81.8 & 83.0 & 80.5 & 84.8 & 82.6 \\
\hline Horse & 368 & 22 & 2 & 82.6 & 82.1 & N/A & 82.6 & 86.7 & 86.0 \\
\hline Hypo & 3163 & 25 & 2 & 99.2 & 98.9 & N/A & 98.4 & 99.4 & 99.4 \\
\hline Iono & 351 & 34 & 2 & 90.0 & 92.3 & 90.0 & 91.5 & 94.3 & 92.5 \\
\hline Iris & 150 & 4 & 3 & 95.3 & 94.7 & 94.7 & 94.0 & 94.7 & 94.7 \\
\hline Labor & 57 & 16 & 2 & 79.3 & 86.3 & N/A & 89.7 & 90.0 & 86.3 \\
\hline Led7 & 3200 & 7 & 10 & 73.5 & 71.9 & N/A & 72.5 & 74.3 & 73.8 \\
\hline Lymph & 148 & 18 & 4 & 73.5 & 77.8 & N/A & 83.1 & 90.0 & 90.0 \\
\hline Pima & 768 & 8 & 2 & 75.5 & 72.9 & 75.0 & 75.1 & 78.4 & 76.2 \\
\hline Sick & 2800 & 29 & 2 & 98.5 & 97.0 & N/A & 97.5 & 97.9 & 97.3 \\
\hline Sonar & 208 & 60 & 2 & 70.2 & 77.5 & N/A & 79.4 & 90.0 & 88.6 \\
\hline Tic-tac-toe & 958 & 9 & 2 & 99.4 & 99.6 & 99.1 & 99.2 & 99.2 & 99.1 \\
\hline Vehicle & 846 & 18 & 4 & 72.6 & 68.7 & 66.3 & 68.8 & 76.3 & 74.9 \\
\hline Waveform & 5000 & 21 & 3 & 78.1 & 80.0 & 84.7 & 83.2 & 87.3 & 85.5 \\
\hline Wine & 178 & 13 & 3 & 92.7 & 95.0 & 97.1 & 95.0 & 98.3 & 98.3 \\
\hline Zoo & 101 & 16 & 7 & 92.2 & 96.8 & N/A & 97.1 & 97.1 & 97.1 \\
\hline \hline Average & & & & $83.34 \%$ & $84.69 \%$ & N/A & $85.22 \%$ & $\mathbf{8 8 . 3 9 \%}$ & $87.36 \%$ \\
\hline
\end{tabular}

taken from [3]. The last column is the accuracy of MEPAR. In this data group, MEPAR performed better than the others on 7 of 13 datasets. It also performed well on both large (Nursery, Mushroom, Splice) and small ones (Lenses, Lungcancer). The average accuracy of MEPAR is significantly higher than those of the others.

we also analyzed the stability of MEPAR. Figure 1 shows a comparison of the log-likelihood and the accuracy between two cases: normal maxent (without PARs) and MEPAR (with PARs). The left graph shows that the log-likelihood of MEPAR increases more strongly than that of the normal maxent. This means that MEPAR can describe the empirical data better. The right graph depicts the accuracy values of MEPAR and the normal maxent as functions of the number of training iterations. As we can see, the accuracy of MEPAR is significantly higher than that of the normal maxent. Further, the accuracy of MEPAR increases smoothly, without large fluctuations of the normal maxent. 
Table 2. Accuracy comparison: ART, C4.5, RIPPER, CN2, N. Bayes, and MEPAR

\begin{tabular}{|l|r|r|r|c|c|c|c|c|c|}
\hline Datasets & \#Rec & \#Attr & \#Cls & ART & C4.5 & RIPPER & CN2 & N.Bayes & MEPAR \\
\hline \hline Audiology & 226 & 69 & 24 & 65.2 & 81.4 & 73.9 & 75.6 & 23.4 & 84.1 \\
\hline Car & 1728 & 6 & 4 & 98.6 & 92.9 & 78.4 & 93.9 & 70.0 & 97.0 \\
\hline Chess & 3198 & 35 & 2 & 97.7 & 99.2 & 99.2 & 99.4 & 62.5 & 96.3 \\
\hline Hayes-roth & 160 & 4 & 3 & 84.4 & 73.8 & 78.1 & 76.2 & 61.9 & 87.5 \\
\hline Lenses & 24 & 5 & 3 & 70.0 & 81.7 & 65.0 & 76.7 & 63.3 & 80.0 \\
\hline Lung-cancer & 32 & 56 & 3 & 40.8 & 43.3 & 45.0 & 39.2 & 43.3 & 53.3 \\
\hline Mushrooms & 8124 & 22 & 2 & 98.5 & 100. & 100. & 100. & 94.3 & 100. \\
\hline Nursery & 12960 & 8 & 5 & 99.1 & 96.2 & 96.7 & 98.1 & 88.0 & 98.5 \\
\hline Soybean & 683 & 35 & 19 & 91.5 & 93.7 & 91.1 & 92.1 & 58.7 & 92.8 \\
\hline Splice & 3190 & 60 & 3 & 89.3 & 94.1 & 93.1 & 92.3 & 51.9 & 96.1 \\
\hline Tic-tac-toe & 958 & 9 & 2 & 81.6 & 83.8 & 97.5 & 98.0 & 65.3 & 99.2 \\
\hline Titanic & 2201 & 3 & 2 & 78.6 & 79.1 & 78.3 & 75.8 & 68.0 & 78.1 \\
\hline Vote & 435 & 16 & 2 & 95.9 & 95.9 & 94.9 & 94.7 & 89.2 & 97.2 \\
\hline \hline Average & & & & $83.94 \%$ & $85.76 \%$ & $83.94 \%$ & $85.54 \%$ & $64.61 \%$ & $\mathbf{8 9 . 2 4 \%}$ \\
\hline
\end{tabular}

$1 \quad 5 \quad 913172125293337414549$

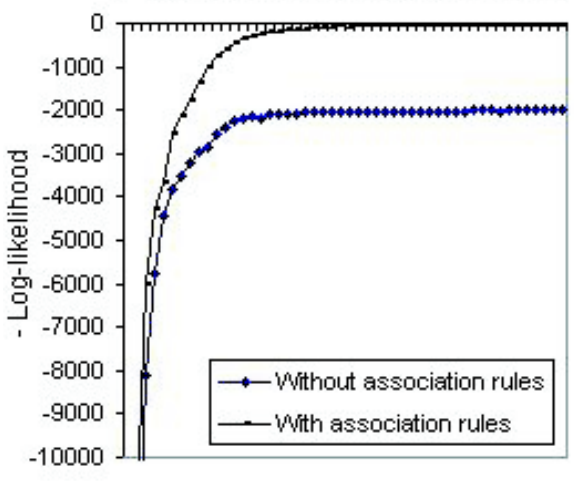

\# of L-BFGS training iterations

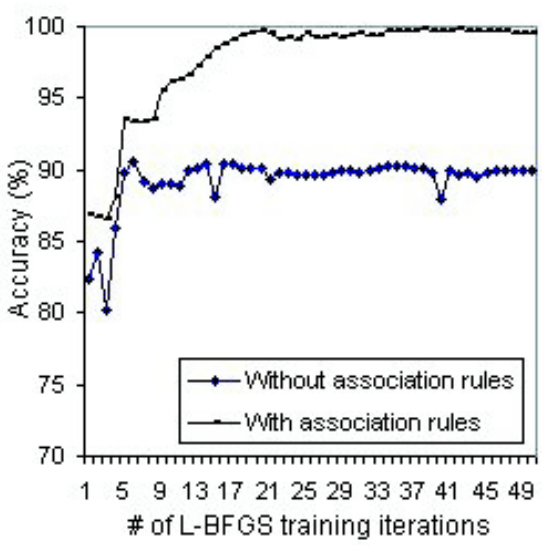

Fig. 1. Log-likelihood and accuracy as functions of \# of L-BFGS training iterations (with and without PARs) - measured on the Nursery dataset at the $1^{\text {st }}$ fold of $10-\mathrm{CV}$

\section{Conclusions}

This paper presented the hybrid classification model, MEPAR, that is based on PARs discovered from training data and maxent. Unlike other rule-based methods, our model predicts labels for new data instances based on multiple PARs whose weights are trained using maxent. This ensures that weights of PARs not only precisely measure how important the PARs are, but are also consistent with the empirical data. Also, a prediction based on multiple PARs should avoid 
biased decisions. Our future work will focus on how to tune parameters automatically in order to obtain higher performance. Also, the theoretical aspect will be further investigated to clarify why MEPAR can achieve such high accuracy.

\section{References}

1. Agrawal, R. and Srikant, R. (1994). Fast algorithms for mining association rules. In Proceedings of VLDB, 487-499.

2. Berger, A., Della Pietra, S., and Della Pietra V. (1996). A maximum entropy approach to natural language processing. Computational Linguistics, 22(1):39-71.

3. Berzal, F., Cubero, J., Sanchez, D., and Serrano, J. (2004). ART: A hybrid classification model. Machine Learning, 54:67-92.

4. Borthwick, A. (1999). A maximum entropy approach to named entity recognition. PhD dissertation, Dept. of CS, New York University.

5. Clark, P. and Nibblett, T. (1989). The CN2 induction algorithm. Machine Learning, 3(4):261-283.

6. Cohen, W. (1995). Fast effective rule induction. In Proceedings of ICML, 115-123.

7. Darroch, N. and Ratcliff, D. (1972). Generalized iterative scaling for log-linear models. The Annals of Mathematical Statistics, 43:1470-1480.

8. Dong, G. and Li, J. (1999). Efficient mining of emerging patterns: discovering trends and differences. In Proceedings of ACM SIGKDD.

9. Dong, G., Zhang, X., Wong, L., and Li, J. (1999). CAEP: Classification by aggregating emerging patterns. In Proceedings of Discovery Science.

10. Duda, R., Hart, P., and Stork, D. (2000). Pattern Classification. Wiley Interscience.

11. Fayyad, M. and Irani, B. (1993). Multi-interval discretization of continuous-valued attributes for classification learning. In Proceedings of IJCAI, 1022-1027.

12. Kohavi, R. and Sahami, M. (1996). Error-based and entropy-based discretization of continuous features. In Proceedings of ACM SIGKDD.

13. Kohavi, R., John, G., Long, R., Manley, D., and Pfleger, K. (1994). MLC++: a machine learning library in C++. In Tools with artificial intelligence, 740-743.

14. Han, J., Pei, J., Yin, Y. (2000). Mining frequent patterns without candidate generation. In Proceedings of ACM SIGMOD, 1-12.

15. Hettich, S., Blake, L., and Merz, J. (1998). UCI Repository of machine learning databases. http://www.ics.uci.edu/ mlearn/MLRepository.html. Irvine, CA: University of California, Department of Information and Computer Science.

16. Liu, B., Hsu, W., and Ma, Y. (1998). Integrating classification and association rule mining. In Proceedings of ACM SIGKDD.

17. Li, W., Han, J., and Pei, J. (2001). CMAR: Accurate and efficient classification based on multiple class-association rules. In Proceedings of IEEE ICDM.

18. Liu, D. and Nocedal, J. (1989). On the limited memory BFGS method for largescale optimization. Mathematical Programming, 45:502-528.

19. Malouf, R. (2002). A comparison of algorithms for maximum entropy parameter estimation. In Proceedings of CoNLL.

20. Pietra, S., Pietra, V., and Lafferty, J. (1997). Inducing features of random fields. IEEE Transactions on Pattern Analysis and Machine Intelligence, 19(4):380-393.

21. Quinland, J. (1993). C4.5: Programs for machine learning. Morgan Kaufmann.

22. Ratnapharkhi, A. (1996). A maximum entropy model for part-of-speech tagging. In Proceedings of EMNLP. 